\title{
Studying Effects of Transcranial Alternating Current Stimulation on Hearing and Auditory Scene Analysis
}

\author{
Lars Riecke
}

\begin{abstract}
Recent studies have shown that perceptual detection of near-threshold auditory events may depend on the relative timing of the event and ongoing brain oscillations. Furthermore, transcranial alternating current stimulation (tACS), a non-invasive and silent brain stimulation technique, can entrain cortical alpha oscillations and thereby provide some experimental control over their timing. The present research investigates the potential of delta/theta-tACS to modulate hearing and auditory scene analysis. Detection of near-threshold auditory stimuli, which are modulated at $4 \mathrm{~Hz}$ and presented at various moments (phase lags) during ongoing tACS (two synchronous $4-\mathrm{Hz}$ alternating currents applied transcranially to the two cerebral hemispheres), is measured in silence or in a masker. Results indicate that performance fluctuates as a function of phase lag and these fluctuations can be explained best by a sinusoid at the tACS frequency. This suggests that tACS may amplify/attenuate sounds that are temporally coherent/anticoherent with tACSentrained cortical oscillations.
\end{abstract}

Keywords Brain stimulation · Neural oscillation · Entrainment · Auditory cortex • Phase alignment

\section{Introduction}

\subsection{What Is TACS?}

TACS is a non-invasive brain stimulation technique that can be applied safely and silently using portable and relatively inexpensive equipment. Its application involves inducing a weak electric current between electrodes at the scalp (Nitsche et al. 2008; Zaghi et al. 2010; Paulus 2011). The current partially penetrates the brain, where it propagates widely and forces neuronal excitability to oscillate along with its alternating waveform (Frohlich and McCormick 2010; Ozen et al. 2010).

\footnotetext{
L. Riecke $(\bowtie)$

Department of Cognitive Neuroscience, Maastricht University, Maastricht, The Netherlands e-mail: L.Riecke@MaastrichtUniversity.NL 
In this way, tACS may entrain ongoing cortical oscillations at corresponding frequencies and impact on different aspects of sensory-perceptual-motor processing and higher-order cognition, depending on stimulation parameters such as electrode positions (reviewed in Herrmann et al. 2013).

\subsection{Cortical Oscillatory Phase Influences Auditory Perception}

Cortical oscillations may also entrain with periodic auditory stimuli, so that high neuronal excitability phases become aligned with peaks in the sound envelope. This sound-brain entrainment is thought to facilitate cortical processing of upcoming acoustic events that concur with sound envelope peaks (Schroeder and Lakatos 2009; Arnal and Giraud 2012). While correlational studies showed that sound-brain entrainment is involved in the neural processing and perception of auditory stimuli (Stefanics et al. 2010; Besle et al. 2011; Gomez-Ramirez et al. 2011), only three published human studies sought to directly control this entrainment with periodic external stimulation and assess the perceptual effects. Neuling et al. (2012) presented tone pips in noise and simultaneously applied 10-Hz tACS above the auditory cortices while manipulating the relative timing of the tone and ACS (i.e., the phase lag). They found that detection performance varied as a function of phase lag and that this function resembled well the tACS waveform. Henry (2012; Henry et al. 2014) used a periodic 3- or 5-Hz modulation applied to an interrupted tone carrier as entraining stimulus. They manipulated the relative timing of the gap and the ongoing modulation and found that this influences gap detection in the phase-dependent manner described above for the tACS study. Based on electrophysiological recordings (Lakatos et al. 2005; Frohlich and McCormick 2010; Ozen et al. 2010; Ali et al. 2013), it appears plausible that the reported effects on auditory perception were mediated by auditory cortical oscillations that entrained to the periodic external stimulation and thereby caused the auditory targets to arrive at different neural excitability phases.

\subsection{Can TACS Influence Auditory Perception?}

Compared with periodic acoustic stimulation, tACS arguably provides the preferred entrainment stimulus as it can be applied both in silence and at selected scalp position, thus allowing to bypass the peripheral auditory system and reduce possible masking of the target sound. However, important issues remain unresolved: How reliable are auditory effects of tACS? Do they also apply to oscillations outside the alpha range? Do they also occur in more naturalistic situations where target sounds need to be tracked amidst other sounds?

This manuscript provides preliminary answers to these questions based on ongoing tACS research. A first experiment (Riecke et al. 2015) shows that auditory 
effects of alpha-tACS phase (Neuling et al. 2012) can be reproduced for the delta/ theta range using a more rigorous methodological approach. An ongoing follow-up experiment is using an informational masking paradigm to explore whether these results can be extended to sustained sounds in scenes.

\section{Methods}

\subsection{Stimuli}

\subsubsection{TACS}

To facilitate homophasic stimulation in both auditory cortices (target regions), two approximately equivalent circuits were generated using two stimulator systems (Neuroconn, Ilmenau, Germany): Prior electric field simulations suggested that placing the stimulation electrodes at position $\mathrm{T} 7$ and $\mathrm{T} 8$, and the return electrodes close to position $\mathrm{Cz}$, would produce relatively strong currents in the target regions. The same sinusoidal current (frequency: $4 \mathrm{~Hz}$, starting phase: $0^{\circ}$ ) was applied to each lateralized circuit, the return electrodes were coupled, and the skin was prepared so that the left- and right-lateralized circuit impedances were matched, while keeping the net impedance below $10 \mathrm{k} \Omega$. Current strength was set individually to the point where participants reported being comfortable with receiving tACS under all electrodes.

\subsubsection{Auditory Stimuli}

Target sounds were modulated at the tACS frequency $(4 \mathrm{~Hz})$ to give listeners the opportunity to sample the target at fixed phase angle on consecutive tACS cycles. The relative level of target and background was set individually to a value near detection threshold determined beforehand.

In experiment 1, the target was a click train. It was generated by summing all harmonics (sinusoids with fixed amplitude and starting phase) of a 4-Hz fundamental within the range from 112 to $3976 \mathrm{~Hz}$ and bandpass-filtering the resulting waveform between 224 and $1988 \mathrm{~Hz}$. The portion from 125 to $1125 \mathrm{~ms}$ was extracted to obtain four clicks centered on a 1-s interval.

In experiment 2, the target was a pulsed complex tone embedded in a masker. The target was generated by summing three sinusoidal harmonics of a $110-\mathrm{Hz}$ fundamental that were chosen randomly from the range from 220 to $6270 \mathrm{~Hz}$ with the constraint that they differed by at least one critical band. The target was modulated with a square wave function (frequency: $4 \mathrm{~Hz}$, duty cycle: $41.7 \mathrm{~ms}$ ) and its duration was chosen randomly from the interval from 2 to $6 \mathrm{~s}$. The masker was a continuous sequence of random complex tones, each of which was generated by summing three to five sinusoids (depending on the listener's target detection threshold) with 
random starting phases and the same amplitude and duration as the target components. The masker components were chosen randomly from the range from 200 to $6297 \mathrm{~Hz}$ with the constraint that they differed by at least one critical band from each other and the target components when the latter were present. For intervals when the target was present, overall amplitude cues were eliminated by reducing the number of masker components by the number of target components.

\subsection{Design and Task}

The critical parameter was the relative timing of acoustic and electric stimulation (Fig. 1a). This phase lag was manipulated across six conditions by varying the onset of the target in steps of $41.7 \mathrm{~ms}\left(30^{\circ}\right)$ across the 4-Hz tACS cycles. Each experiment lasted $10 \mathrm{~min}$. It was presented four times during continuous tACS and once without tACS. The latter sham stimulation was identical to tACS, except that the current was ramped down/up shortly after/before the experiment began/ended (Herrmann et al. 2013). Debriefings revealed that participants were unaware of whether they received tACS or sham stimulation. The order of trials and stimulation type (tACS, sham) was randomized individually.

Fig. 1 Experimental designs. Panel A illustrates the six tACS phase conditions, each of which was characterized by a specific phase lag between the acoustic and electric stimuli. Gray waves schematize four consecutive 4-Hz tACS cycles. Black dashes sketch the target sound, which was either a 4-Hz click train (experiment 1) or a harmonic complex pulsed at $4 \mathrm{~Hz}$ (experiment 2). Panel B exemplifies the visual, electric, and auditory stimulation, and a behavioral response from a trial in experiment 1. Listeners detected clicks in a $2 \mathrm{I} 2 \mathrm{AFC}$ task. Analogous to panel B, panel $\mathrm{C}$ exemplifies a portion of a trial from experiment 2 . Listeners held a bar whenever they heard a repetitive tone, which occurred at unpredictable times within an ongoing multi-tone masker

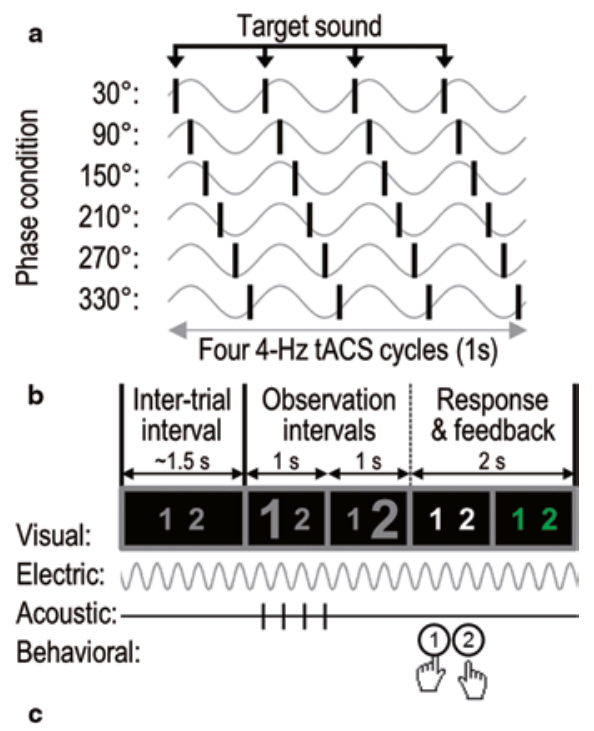

Electric: WWWWWWWMWM

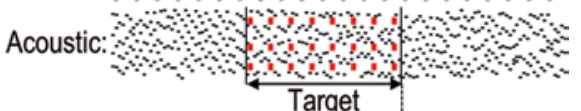

Behavioral:

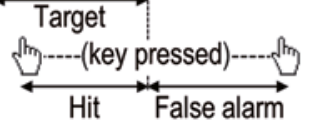


In experiment 1 , click detection performance was assessed using a two-interval two-alternative forced-choice task (2I2AFC); see Fig. 1b. The click train was presented in a randomly selected interval, whereas the other interval contained only silence. Listeners judged which interval contained the clicks and received visual feedback. Visual stimulus timing was jittered to provide no valid cue of the click timing.

In experiment 2 (Fig. 1c), target detection was assessed using a continuous yes/ no task. On each trial, the masker was presented for $45 \mathrm{~s}$ during which several targets were presented. Consecutive targets were separated by non-target intervals whose duration varied randomly between 2 and $7 \mathrm{~s}$, with the constraint that their overall duration matched that of the target intervals. Trials began and ended with non-target intervals. Listeners were instructed to press and hold a bar as soon and as long as they heard a repetitive tone.

\subsection{Data Analysis}

A performance measure was extracted for each tACS phase condition: For experiment 1 , accuracy was extracted, defined as the number of correct responses divided by the number of trials. In experiment 2 , the sensitivity index d' was computed based on the overall time during which the participant correctly or erroneously reported the target, divided by the overall target or non-target time (respectively).

Next, a 'time series' of the performance measure was reconstructed by concatenating the six phase conditions. To compensate for possible inter-individual variations in the phase for which performance was best, the maximum of the series was aligned to the $90^{\circ}$-point and the series was phase-wrapped (e.g., $\mathrm{Ng}$ et al. 2012). Following this 'best-phase alignment' - under the main hypothesis that 4-Hz tACS phase modulates detection - phases $30-150^{\circ}$ should delimit a positive $4-\mathrm{Hz}$ halfcycle, whereas phases $210-330^{\circ}$ should delimit the opposite (negative) half-cycle. To verify this prediction, performance was averaged across the hypothesized positive half-cycle (phases 30 and $150^{\circ}$ ) and the opposite half-cycle (phases 210, 270, and $330^{\circ}$ ), and the two resulting averages were compared. The alignment point $\left(90^{\circ}\right)$ was excluded to preserve statistical independency. To verify whether the hypothesized phase effect cycled specifically at $4 \mathrm{~Hz}$, spectral density was computed from the series and the resulting frequency components were compared. Only three bins centered on 4,8 , and $12 \mathrm{~Hz}$ could be resolved, due to the limited number of data points and sampling rate (six points spanning a $4-\mathrm{Hz}$ period). Baseline was defined as the overall performance during the sham run, excluding trials presented during the tACS ramps. 


\subsection{Participants}

Fourteen and eleven paid volunteers (ten females, ages: 20-38 years) participated in experiment 1 and experiment 2 so far. They reported no history of neurological, psychiatric, or hearing disorders, were eligible to undergo tACS as assessed by prior screening, and gave their written informed consent before taking part. They had normal audiometric thresholds, except for one participant with mild high-frequency hearing loss.

\section{Results}

Figure 2a shows listeners' average performance in experiment 1 as a function of phase condition. Performance was above baseline in the 30 and $150^{\circ}$ conditions, whereas it was below baseline in the 210,270 , and $330^{\circ}$ conditions. Furthermore, performance was similar at 30 and $150^{\circ}$, and at 210 and $330^{\circ}$ respectively, i.e.,
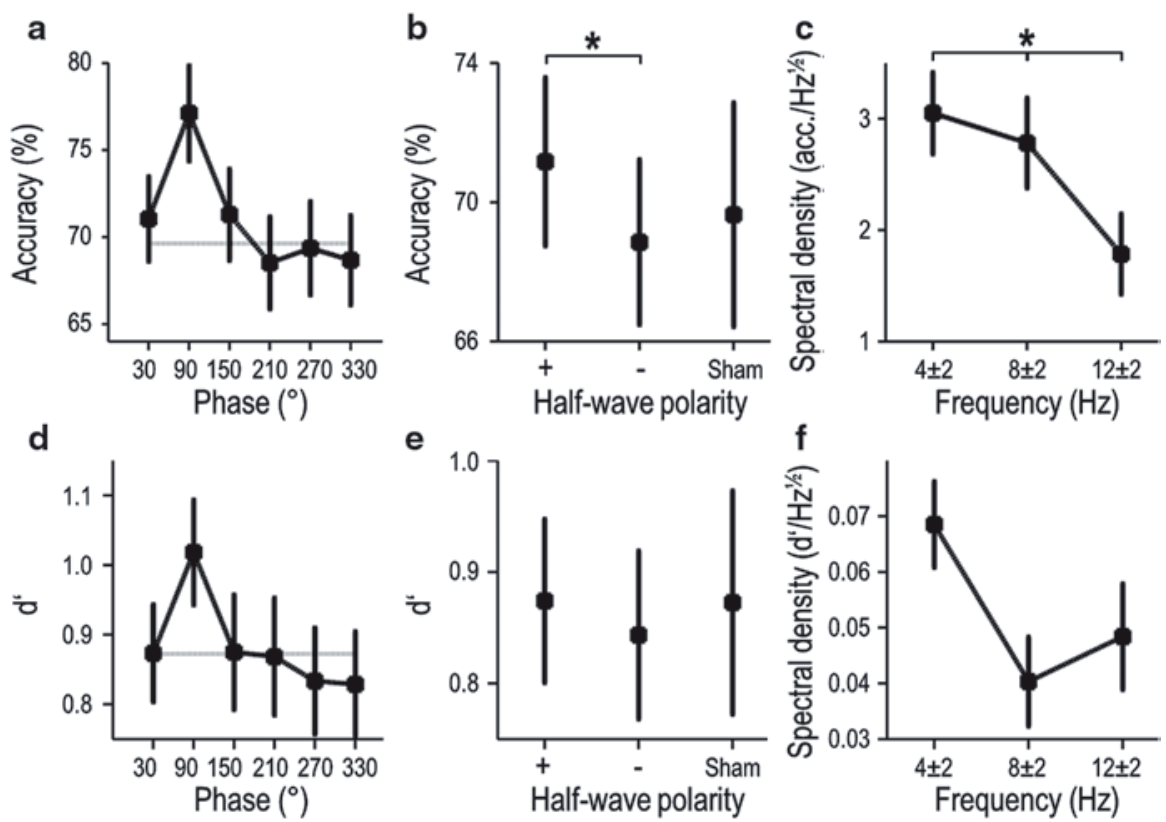

Fig. 2 Results. Panel A shows detection performance (mean \pm sem across listeners) as a function of phase condition in experiment 1 . The gray horizontal line shows performance under sham stimulation. Panel B shows averages of these data, i.e., the hypothesized positive half-cycle (average of conditions 30 and $150^{\circ}$ ), the opposite half-cycle (average of conditions 210,270 , and $330^{\circ}$ ), and sham stimulation. Panel C shows the magnitude spectrum of the performance waveform (mean \pm sem across listeners' individual magnitude spectra). Analogously, panels D, E, and F show preliminary data from experiment 2 
it was approximately symmetric with respect to the presumed best phase $\left(90^{\circ}\right)$. Although on average the best and worst performances were not associated with exactly opposite phases, possibly due to the contribution of higher harmonics, these observations match well the characteristics of the hypothesized 4-Hz cycle.

Figure $2 \mathrm{~b}$ shows performance averaged across phase conditions to facilitate comparison of the hypothesized positive half-cycle $\left(30,150^{\circ}\right)$ with the opposite half-cycle $\left(210,270,330^{\circ}\right)$ and baseline. Paired t-tests revealed an effect of halfcycle polarity on performance (positive vs. negative half-cycle: $t(13)=2.48$, corrected $p=0.042$ ); thus tACS phase modulated click detection. Comparison with baseline revealed no effect.

Figure $2 \mathrm{c}$ shows the magnitude spectrum of the series. While frequency bins centered on the higher harmonics $(8$ and $12 \mathrm{~Hz}$ ) could explain a significant proportion of the overall variance in the series, this variance was explained best by a 4-Hz sinusoid; thus the phase-induced differences cycled predominantly at the tACS frequency. This observation was supported by a random-effects ANOVA including frequency as factor, which revealed a main effect of frequency on spectral magnitude $(\mathrm{F}(2,26)=3.93, p=0.032)$. Post-hoc paired t-tests showed that the $4-\mathrm{Hz}$ bin was significantly stronger than the $12-\mathrm{Hz}$ bin $(\mathrm{t}(13)=2.65$, corrected $p=0.020)$ but not the 8-Hz bin.

Analogously, Fig. 2d, f show preliminary results from experiment 2 based on d'. These data are shown at the descriptive level given the limited sample size. As for experiment 1 , performance appears to be on average slightly better during the hypothesized positive 4-Hz half-cycle than during the opposite half-cycle (Fig. 2d, e) and the magnitude spectrum shows a peak at the tACS frequency (Fig. 2f). Statistical analyses based on a larger sample, which is being acquired at the moment, will assess enable to assess the significance of these preliminary observations.

\section{Discussion}

Experiment 1 revealed that click detection under 4-Hz tACS is modulated by tACS phase: performance was significantly better during one half of the 4-Hz tACS cycle than during the opposite half. Performance without tACS was intermediate, but did not differ significantly. The tACS phase effect was strongest in the vicinity of the tACS frequency but not strictly frequency-specific as it extended to higher frequencies. Overall, these data replicate the findings by Neuling et al. (2012), indicating that auditory effects of tACS phase are reproducible. They extend these previous findings, which were based on single tone pips and alpha-tACS, to new tasks and stimuli including periodic clicks and dual-channel delta/theta-tACS. Considering that clicks were presented at the tACS frequency and their detectability was found to depend on tACS phase, a possible interpretation is that tACS essentially enhanced the loudness of those click trains that were temporally coherent (rather than anti-coherent) with tACS-entrained oscillations. Such an 'envelope enhancement' would have interesting implications for auditory scene analysis problems that benefit from tracking the envelope of the target sound. 
This idea is being addressed in experiment 2, where an otherwise audible modulated target sound is rendered inaudible by spectrally non-overlapping distractor sounds. Based on magnetoencephalography findings from a similar paradigm, Elhilali et al. (2009) proposed that the perceptual segregation of the modulated target from the distractors arises from temporal coherence of cortical activity patterns with the target. Whether tACS phase modulates this temporal coherence and therewith the segregability of the target remains to be shown; the preliminary data presented here indicate a trend toward a possible effect of 4-Hz tACS phase, as in experiment 1.

Observations of actual benefits of tACS compared with sham stimulation are rather modest so far. In experiment 1, click detection improved on average by $7 \%$ (at best phase), a difference that did not reach statistical significance. Neuling et al. (2012) observed a 0.3-dB improvement in tone detection. Future studies may identify which tACS parameters (e.g., single or dual channel tACS, 4 or $10 \mathrm{~Hz}$ frequency) are most effective and systematically optimize them on an individual basis in order to maximize possible perceptual benefits. For example, the tACS frequency could be chosen to match the individual's dominant ongoing cortical frequency as identified beforehand with EEG (Zaehle et al. 2010; Ali et al. 2013). Similarly, tACS electrode positions could be chosen based on more realistic current flow simulations using as head model the individual's anatomy obtained beforehand with MRI (e.g., (Wagner et al. 2014)). Due to the limited spatial specificity of transcranial stimulation, individual tonotopic locations cannot be selectively targeted. Nevertheless, the possibility to transmit sound envelope information non-invasively and bypass (possible deficits in) the auditory pathway up to the cortex might make transcranial stimulation a valuable tool.

Acknowledgments I would like to thank Alexander Sack, Daniel Hauke, and Marijn van Waardenburg for help with obtaining the data. This work was supported by NWO Veni grant 451-11-014. Some of the data presented here have been published elsewhere (Riecke et al. 2015).

Open Access This chapter is distributed under the terms of the Creative Commons AttributionNoncommercial 2.5 License (http://creativecommons.org/licenses/by-nc/2.5/) which permits any noncommercial use, distribution, and reproduction in any medium, provided the original author(s) and source are credited.

The images or other third party material in this chapter are included in the work's Creative Commons license, unless indicated otherwise in the credit line; if such material is not included in the work's Creative Commons license and the respective action is not permitted by statutory regulation, users will need to obtain permission from the license holder to duplicate, adapt or reproduce the material.

\section{References}

Ali MM, Sellers KK, Frohlich F (2013) Transcranial alternating current stimulation modulates large-scale cortical network activity by network resonance. J Neurosci 33(27):11262-11275

Arnal LH, Giraud AL (2012) Cortical oscillations and sensory predictions. Trends Cogn Sci 16(7):390-398 
Besle J, Schevon CA, Mehta AD, Lakatos P, Goodman RR, McKhann GM, Emerson RG, Schroeder CE (2011) Tuning of the human neocortex to the temporal dynamics of attended events. J Neurosci 31(9):3176-3185

Elhilali M, Xiang J, Shamma SA, Simon JZ (2009) Interaction between attention and bottom-up saliency mediates the representation of foreground and background in an auditory scene. PLoS Biol 7(6):e1000129

Frohlich F, McCormick DA (2010) Endogenous electric fields may guide neocortical network activity. Neuron 67(1):129-143

Gomez-Ramirez M, Kelly SP, Molholm S, Sehatpour P, Schwartz TH, Foxe JJ (2011) Oscillatory sensory selection mechanisms during intersensory attention to rhythmic auditory and visual inputs: a human electrocorticographic investigation. J Neurosci 31(50):18556-18567

Henry MJ, Obleser J (2012) Frequency modulation entrains slow neural oscillations and optimizes human listening behavior. Proc Natl Acad Sci U S A 109(49):20095-20100

Henry MJ, Herrmann B, Obleser J (2014) Entrained neural oscillations in multiple frequency bands comodulate behavior. Proc Natl Acad Sci U S A 111(41):14935-14940

Herrmann CS, Rach S, Neuling T, Struber D (2013) Transcranial alternating current stimulation: a review of the underlying mechanisms and modulation of cognitive processes. Front Hum Neurosci 7:279

Lakatos P, Shah AS, Knuth KH, Ulbert I, Karmos G, Schroeder CE (2005) An oscillatory hierarchy controlling neuronal excitability and stimulus processing in the auditory cortex. J Neurophysiol 94(3):1904-1911

Neuling T, Rach S, Wagner S, Wolters CH, Herrmann CS (2012) Good vibrations: oscillatory phase shapes perception. Neuroimage 63(2):771-778

Ng BS, Schroeder T, Kayser C (2012) A precluding but not ensuring role of entrained low-frequency oscillations for auditory perception. J Neurosci 32(35):12268-12276

Nitsche MA, Cohen LG, Wassermann EM, Priori A, Lang N, Antal A, Paulus W, Hummel F, Boggio PS, Fregni F, Pascual-Leone A (2008) Transcranial direct current stimulation: state of the art 2008. Brain Stimul 1(3):206-223

Ozen S, Sirota A, Belluscio MA, Anastassiou CA, Stark E, Koch C, Buzsaki G (2010) Transcranial electric stimulation entrains cortical neuronal populations in rats. J Neurosci 30(34):1147611485

Paulus W (2011) Transcranial electrical stimulation (tES - tDCS; tRNS, tACS) methods. Neuropsychol Rehabil 21(5):602-617

Riecke L, Formisano E, Herrmann CS, Sack AT (2015). 4-Hz transcranial alternating current stimulation phase modulates hearing. Brain Stimul 8:777-783

Schroeder CE, Lakatos P (2009) Low-frequency neuronal oscillations as instruments of sensory selection. Trends Neurosci 32(1):9-18

Stefanics G, Hangya B, Hernadi I, Winkler I, Lakatos P, Ulbert I (2010) Phase entrainment of human delta oscillations can mediate the effects of expectation on reaction speed. J Neurosci 30(41):13578-13585

Wagner S, Rampersad SM, Aydin U, Vorwerk J, Oostendorp TF, Neuling T, Herrmann CS, Stegeman DF, Wolters CH (2014) Investigation of tDCS volume conduction effects in a highly realistic head model. J Neural Eng 11(1):016002

Zaehle T, Rach S, Herrmann CS (2010) Transcranial alternating current stimulation enhances individual alpha activity in human EEG. PLoS ONE 5(11):e13766

Zaghi S, Acar M, Hultgren B, Boggio PS, Fregni F (2010) Noninvasive brain stimulation with lowintensity electrical currents: putative mechanisms of action for direct and alternating current stimulation. Neuroscientist 16(3):285-307 